\section{Privatklinik Wyss}

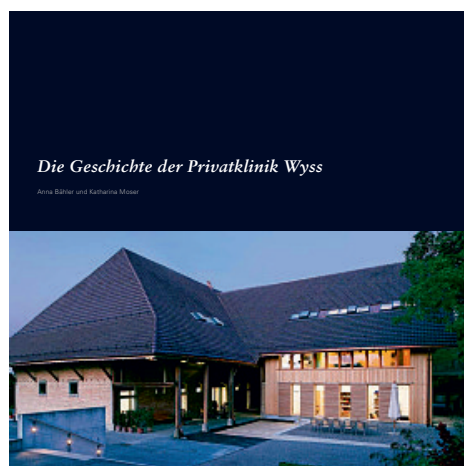

Anna Bähler, Katharina Moser

Die Geschichte der Privatklinik Wyss

Bern: Eigenverlag; 2010.

96 Seiten. 30 CHF.

(Zu beziehen über die Klinik

oder die Buchhandlung Stauffacher, Bern.)

Die ansprechende «Firmengeschichte» soll das 165 . Jubiläum, vor allem aber ein neues Qualitätsbewusstsein der Privatklinik in Münchenbuchsee feiern. Zwei Historikerinnen haben deren Geschichte aufgrund von Materialien aller Art erarbeitet. Ihrem impliziten Auftrag, zum Lob der Institution zu schreiben, konnten sie mit Leichtigkeit genügen: Die Geschichte der Klinik Wyss ist tatsächlich die eines bemerkenswerten Familienbetriebs, der sich über sechs Eignergenerationen immer wieder verändert und neu bewährt hat. 1845 hat der von der aufgeklärten Atmosphäre in der Berner Gemeinde Münchenbuchsee angezogene Arzt J. C. Straub die damalige «Anstalt für Gemütskranke» gegründet. Als er 1855 starb, übernahm seine junge Frau Anna, geb. Reber, den Betrieb, vergrösserte ihn beträchtlich und vererbte ihn 1884 ihrer Nichte und Mitarbeiterin Katharina Reber. In die Hände des männlichen Stammes Wyss ging das Werk über, als Katharina den Krankenwärter Rudolf Wyss heiratete. Ein Landwirtschaftsbetrieb war bis in die 1950er Jahre integrierender, auch therapeutisch wichtiger Teil der Institution. Prospekte, die seit Ende des 19. Jahrhunderts periodisch neu gestaltetet wurden, illustrieren die Entwicklungen des Selbstbildes der Klinik.

Seit dem 100-Jahr-Jubiläum, gefeiert mit der Publikation von R. Petitmermets «Geschichte des Nervensanatoriums Wyss», hat sich viel verändert. Die Anstalt wurde «von einer «pflegerischen mehr zu einer therapeutischen Klinik». 1953 begann in Münchenbuchsee mit dem Chlorpromazin die «pharmakologische Wende». Sie brachte einen Aufschwung sozial- und psychotherapeutischer Verfahren mit sich und ein Abrücken von den heroischen Kuren der ersten Jahrhunderthälfte (Malaria- und Schlafkuren, später Schocktherapien). Fenstergitter wurden entfernt und Begegnungen organisiert. Die Pflege zwischenmenschlicher Beziehungen wird als ein Charakteristikum der Wyss'schen Klinik beschrieben. Sie kann auch am seltenen Wechsel der Chefärzte abgelesen werden: «Die Privatklinik Wyss wurde in den letzten 70 Jahren von nur gerade sechs Chefärzten geleitet», schreibt Markus Wyss in seinem Vorwort. Auf Nachhaltigkeit des Betriebs und soziale Verpflichtungen wurde nicht erst in den letzten Jahren geachtet. Seit 1988 gibt es da auch einen kleinen, allgemein zugänglichen Tierpark. Nach einer Krisenzeit in den 1980er Jahren hat sich der Betrieb in vielen Hinsichten neu strukturiert und erweitert, etwa um Ambulatorien in Bern und Biel. Es ist zu hoffen, dass er seine humanitäre, soziale und ökologische Grundhaltung auch als «Firma» in einer neoliberalen Atmosphäre aufrechterhalten kann.

Eine etwas allzu stereotype psychiatriegeschichtliche Einleitung und am Ende einige für die Institution werbende Bilder, einige nützliche Zusammenstellungen sowie Quellenangaben umrahmen die vorgelegte, ansprechende Geschichte der ehrwürdigen Privatklinik Wyss.

Esther Fischer-Homberger, Bern

\section{Älterwerden}

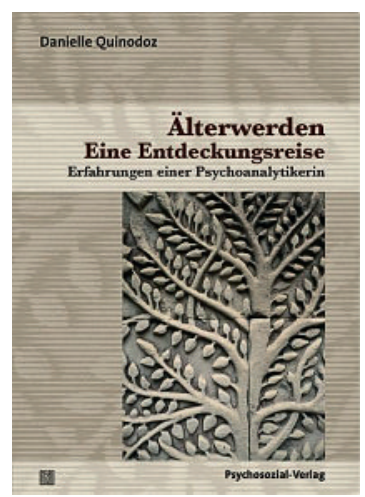

\section{Danielle Quinodoz}

\section{Älterwerden - eine Entdeckungsreise}

Erfahrungen einer Psychoanalytikerin

Gießen: Psychosozial-Verlag; 2010.

230 Seiten. 42.90 CHF.

ISBN 978-3-838379-2012-3

Die Autorin nimmt den Leser, die Leserin mit auf eine Reise in die analytische Arbeit mit ihren Klienten. Sie schildert leicht fassbar mit vielen Fallbeispielen, wie sie die Auseinandersetzung der Klienten mit ihrer eigenen Vergangenheit, ihrer Lebensgeschichte moderiert und begleitet, und führt dabei die Leser in die spezifischen Themen des höheren Lebensalters ein. Dabei zeigt sich, dass dieses Tun vor allem der Verinnerlichung und Integration von belastenden Ereignissen dient zum Zwecke der Versöhnung und der Ermöglichung, das Leben bis zuletzt zu Ende leben zu können. Es geht der Autorin um die Hinwendung zu einer stabilen inneren Welt, die oft auch Defizite im exekutiven Bereich zu kompensieren vermag und damit gelegentlich der Sublimation dient.

Dabei zeigt sich, dass sich die analytische Arbeit mit alten Menschen, obwohl sich der zeitgeschichtliche Kontext, Einstellungen und Werte stark gewandelt haben, nicht grundsätzlich von der analytischen Arbeit mit jüngeren Menschen unterscheidet, dass die Wiederkehr emotionaler Kindheitserfahrungen im Zentrum liegt und die Bearbeitung reaktivierter Konflikte bedingt. Die therapeutische Zielsetzung geht dahin (im Unterschied zu jüngeren Personen), das Gefühl von Unsicherheit in der Begrenzung der zur Verfügung stehenden zeitlichen Reserven des eigenen endlichen Lebens zu akzeptieren und diese begrenzte Spanne Leben mit bestmöglicher Lebensqualität füllen zu können.

Als Therapeutin achtet die Autorin besonders darauf, die äusseren Bedingungen so zu gestalten, dass die Hinwendung zur inneren Welt gelingen kann. Sie kommt aber selber nicht darum herum, sich mit eigenem Altern auseinanderzusetzen, sich mit der eigenen (zeitlichen) Begrenztheit zu versöhnen und durch hingebungsvolle Selbstpflege für die eigene Lebensqualität besorgt zu sein.

Das Buch ist auch für fachfremde Personen leicht lesbar. Gelegentliche Übersetzungsfehler tun ihm wenig Abbruch. Vermissen kann man, dass die zahlreichen Fallgeschichten nicht konsequent mit Altersangaben der betroffenen Menschen versehen sind. Alles in allem aber macht das Buch Lust, sich selber an die analytische-therapeutische Arbeit mit alten Menschen zu wagen. Man wird dabei entdecken, dass diese Auseinandersetzung uns unweigerlich zum Thema unseres eigenen Älterwerdens führt.

Barbara Hiss, Basel 